\title{
Shock wave response of a zirconium-based bulk metallic glass and its composite
}

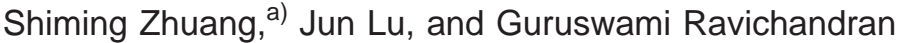 \\ Graduate Aeronautical Laboratories, California Institute of Technology, Pasadena, California 91125
}

(Received 18 February 2002; accepted for publication 24 April 2002)

\begin{abstract}
A zirconium-based bulk metallic glass, $\mathrm{Zr}_{41.2} \mathrm{Ti}_{13.8} \mathrm{Cu}_{12.5} \mathrm{Ni}_{10} \mathrm{Be}_{22.5}$ (Vit 1), and its composite, $\mathrm{Zr}_{56.3} \mathrm{Ti}_{13.8} \mathrm{Cu}_{6.9} \mathrm{Ni}_{5.6} \mathrm{Nb}_{5.0} \mathrm{Be}_{12.5}(\beta$-Vit), were subjected to planar impact loading. A surprisingly low amplitude elastic precursor and bulk wave, corresponding to the elastic response of the "frozen structure" of the intact metallic glasses, were observed to precede the rate-dependent large deformation shock wave. A concave downward curvature after the initial increase of the $U_{s}-U_{p}$ shock Hugoniots suggests that a phase-change-like transition occurred during shock compression. Further, compression damage occurred due to the shear localization. The spalling in Vit 1 was induced by shear localization, while in $\beta$-Vit, it was due to debonding of the $\beta$-phase boundary from the matrix. The spall strengths at strain rate of $2 \times 10^{6} \mathrm{~s}^{-1}$ were determined to be 2.35 and $2.11 \mathrm{GPa}$ for Vit 1 and $\beta$-Vit, respectively. (C) 2002 American Institute of Physics.
\end{abstract}

[DOI: $10.1063 / 1.1485300]$

Metallic glasses are amorphous metastable solids and are being processed in bulk form suitable for structural applications including those involving impact. Due to their noncrystalline, fluid-like randomly ordered atomic structures resulting from the controlled solidification processes from liquid to solid state, metallic glasses have many unique mechanical, electrical, magnetic, and corrosion properties which distinguish them from their counterparts of crystalline alloys. ${ }^{1,2}$

The mechanical properties and structural performance, as well as the deformation and damage mechanisms of bulk metallic glasses have been extensively studied through different techniques, and considerable amount of experimental data and theoretical modeling are available in literature. However, only a few studies if there is any, can the work be found in the literature addressing the dynamic response and damage of metallic glasses under shock loading. In this letter, we report the first shock compression experimental results for a metallic glass, $\mathrm{Zr}_{41.2} \mathrm{Ti}_{13.8} \mathrm{Cu}_{12.5} \mathrm{Ni}_{10} \mathrm{Be}_{22.5}$ (Vitreloy 1$)^{3}$ and its particulate composite, $\mathrm{Zr}_{56.3} \mathrm{Ti}_{13.8} \mathrm{Cu}_{6.9} \mathrm{Ni}_{5.6} \mathrm{Nb}_{5.0} \mathrm{Be}_{12.5}$ (in situ dendritc $\beta$-phase reinforced Vitreloy), ${ }^{4}$ which are to be respectively referred to as Vit 1 and $\beta$-Vit hereafter.

Specimens of $20 \mathrm{~mm}$ diameter were impacted by $36 \mathrm{~mm}$ diameter planar flyers which were accelerated to a desired velocity by a powder gun loading system. ${ }^{5}$ The experimental parameters and mechanical properties of the materials are listed in Tables I and II, respectively. The instance of impact by the flyer on the specimen was detected using electric pins $(\mathrm{EPs})^{5,6}$ flush mounted with the impact surface of specimen. The arrival time of shock wave at the rear surface of specimen was sensed either by EPs which were flush mounted with the rear surface of specimen, or by a time-resolved laser velocity interferometer system, VISAR ${ }^{5,7}$ which provides the particle velocity history. Impactors of different materials, polycarbonate (PC), 6061 aluminum alloy (A1), 304 stainless

a)Electronic mail: shiming@cco.caltech.edu steel (SS), and tungsten (W), were used to achieve different levels of shock loading. The rear surface of the impactors were left free. However, a 7.5-mm-thick polymethylmethacrylate (PMMA) plate was bonded on the rear surface of specimen, which supported the electric pins or served as a window, in case when VISAR was employed.

Figure 1 shows the particle velocity profiles at the specimen/PMMA interface for the two materials impacted by PC and Al flyers. The letters A and B marked next to the wave profiles indicate the arrival instances of elastic precursors and bulk waves. For Vit 1, the precursor and bulk wave speeds estimated based on the shock profile data are about $6 \%-7 \%$ larger than those values obtained by ultrasonic measurements (Table II), while for $\beta$-Vit composite, the data of the two measurements are nearly the same. The letter $\mathrm{C}$ in the figure corresponds to the front of deformation shocks. The long ramping shock front (e.g., PC flyer) decreasing with the increase of shock loading indicates a pronounced rate-dependent process during the shock compression. Under the same loading condition (i.e., Al flyer), the slower initial rising and longer relaxation time of the shock profile indicate that $\beta$-Vit has much larger rate dependence than Vit 1 , which may be attributed to the presence of crystalline bcc $\beta$-phase

TABLE I. Parameters for shock wave experiments.

\begin{tabular}{cccc}
\hline \hline Test No. & ${ }^{\mathrm{a}} D_{f} /(\mathrm{mm})$ & ${ }^{\mathrm{b}} V_{f} /(\mathrm{km} / \mathrm{s})$ & ${ }^{\mathrm{c}} D_{s} /(\mathrm{mm})$ \\
\hline 05 & $\mathrm{PC} / 2.87$ & 0.969 & Vit $1 / 3.71$ \\
02 & $\mathrm{~A} 1 / 1.53$ & 0.544 & Vit $1 / 3.72$ \\
12 & $\mathrm{~S} / 1.47$ & 0.857 & Vit $1 / 3.61$ \\
07 & $\mathrm{~W} / 1.79$ & 1.218 & Vit $1 / 7.38$ \\
08 & $\mathrm{PC} / 2.87$ & 0.976 & $\beta$-Vit $/ 3.32$ \\
09 & $\mathrm{~A} 1 / 1.53$ & 0.586 & $\beta$-Vit $/ 3.36$ \\
10 & $\mathrm{SS} / 1.45$ & 0.840 & $\beta$-Vit $/ 3.38$ \\
11 & $\mathrm{~W} / 1.78$ & 1.182 & $\beta$-Vit $/ 6.71$ \\
\hline \hline
\end{tabular}

${ }^{a}$ Flyer thickness.

${ }^{\mathrm{b}}$ Flyer velocity.

${ }^{\mathrm{c}}$ Specimen thickness. 
TABLE II. Mechanical properties of Vit $1, \beta$-Vit obtained by ultrasonic measurements.

\begin{tabular}{lccccc}
\hline \hline Mater. & ${ }^{\mathrm{a}} \rho /\left(\mathrm{g} / \mathrm{cm}^{3}\right)$ & ${ }^{\mathrm{b}} E /(\mathrm{GPa})$ & ${ }^{\mathrm{c}} G /(\mathrm{GPa})$ & ${ }^{\mathrm{d}} K /(\mathrm{GPa})$ & ${ }^{\mathrm{e}} v$ \\
\hline Vit 1 & 6.00 & 98.6 & 36.4 & 112.7 & 0.35 \\
$\beta$-Vit & 6.21 & 80.0 & 29.1 & 103.9 & 0.37 \\
\hline \hline
\end{tabular}

a Density.

${ }^{\text {b} Y o u n g ' s ~ m o d u l u s . ~}$

'Shear modulus.

${ }^{\mathrm{d} B u l k}$ modulus.

ePoisson's ratio.

in Vit 1 like matrix. ${ }^{4} \mathrm{~A}^{\prime}, \mathrm{B}^{\prime}$, and $\mathrm{C}^{\prime}$ correspond to the arrivals of elastic, bulk, and shock release waves in the shocked states. For both Vit 1 and $\beta$-Vit, the differences between velocities of the elastic precursor and elastic release wave, as well as the differences between velocities of the compressive and release bulk waves, are within $2 \%-3 \%$, which are on the order of the experimental error.

One interpretation for the observed surprisingly low amplitude of Hugoniot elastic limits $\left(\sim 10-10^{2} \mathrm{MPa}\right)$ is that the elastic precursor and bulk waves measured here represent the elastic response of the "frozen structure" of the intact metallic glasses. Since there is virtually no atomic rearrangement during the cooling process of liquids into solids, some amount of "free volume" may have been frozen inside the metallic glasses. Upon shock loading, with the compression of the frozen free volume that is being compressed at very low stress level, the intact frozen structure state is broken and deformation localizes at the initial free volume locations due to stress concentration. Due to initiation and avalanching of this process the shock propagation is slowed down. The small step jumps observed on the deformation shock front for experiments with PC flyers (Fig. 1) are related to the shear localization during shock compression.

Figure 2 shows the experimental shock Hugoniots of Vit 1 and $\beta$-Vit. The concave downward curvature of the $U_{s}-U_{p}$ shock Hugoniots is very similar to those observed on the Hugoniots of materials which experienced a phase change upon being compressed..$^{8,9}$ It is believed that the large reduction of shock velocity in this region is due to the onset of catastrophic internal damage of material within the shock front resulting from the shear localization during compression (see Fig. 3 and discussion later). Such a damage mechanism and its effect on shock waves has been explored in the

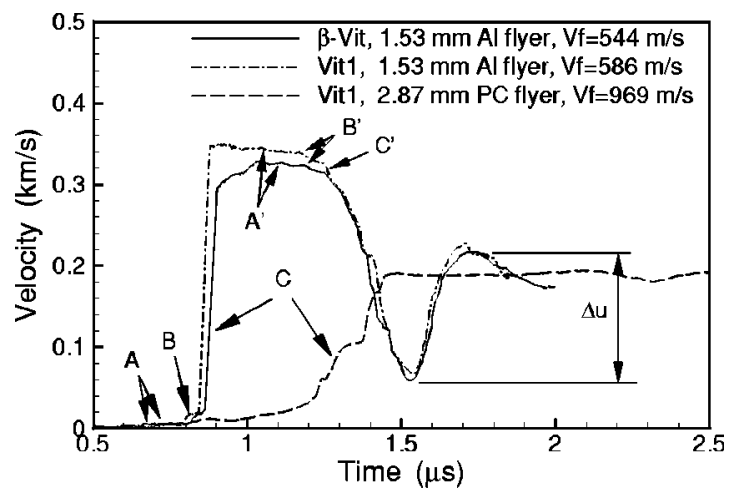

FIG. 1. Particle velocity profiles at the specimen/PMMA interfaces obtained for Vit 1 and $\beta$-Vit specimens impacted by different flyers at various impact velocities. velocities.
Downloaded 12 Jan 2006 to 131.215 .240 .9 . Redistribution subecimen.

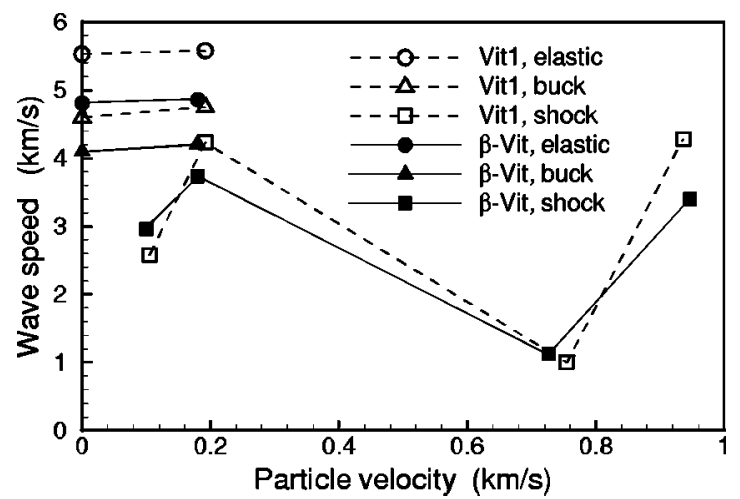

FIG. 2. Shock Hugoniot curves for Vit 1 metallic glass and its particulate composite $\beta$-Vit.

analysis of the formation of failure waves in brittle solids. ${ }^{10}$ The increase in shock velocity with further increase in amplitude of loading is the result of competition between shock compression and relaxation process resulting from shear localization. The elastic and bulk wave speeds for both initial and shocked states estimated using the VISAR velocity profiles are also plotted in Fig. 2. For both Vit 1 and $\beta$-Vit, the deformation shock wave speed is much lower than the bulk wave speed, suggesting the significant reduction in the shear strength at the Hugoniot state. ${ }^{11}$

The recovered specimens were examined using scanning electron microscope (SEM). Microsteps and cracks formed due to shear localization, i.e., shear bands, were observed on the rear surface of the shocked specimens of the two materials as shown in Fig. 3. The bright wavy area around the mature shear bands/cracks was due to the flowing of fluidlike molten material from the localized shear zones, indicating material experienced high temperatures. Through the microstructural examination, it was found that the density (number) of the shear bands/cracks on the rear surface of shocked specimen initially increased with the increasing amplitude of shock loading (by PC, Al, and SS flyers) and reached the maximum under shock loading by SS flyer. However, when the shock loading increased further (using W flyers), the number of shear bands/cracks was found to be decreased, rather than increasing. This observation of trends in damage on the rear surface of shocked specimen correlates with the shock Hugoniot data shown in Fig. 2. This result may be interpreted as damage processes occurring under different shock loading levels were dominated by different physical mechanisms. Under relatively weak loading, though
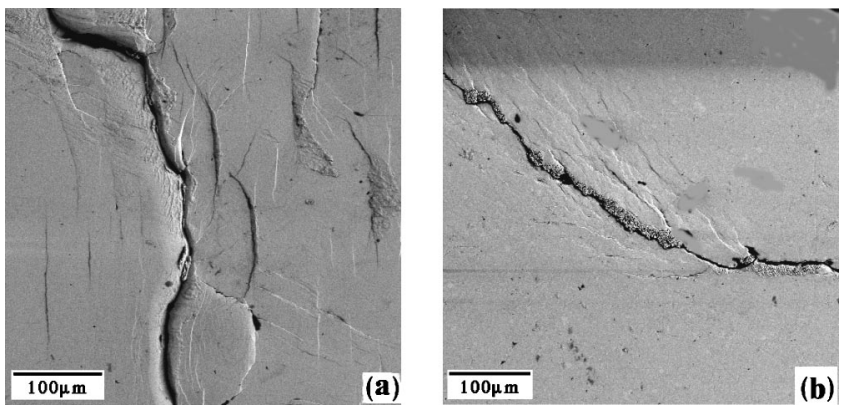

FIG. 3. Typical SEM micrographs of shear bands/cracks observed on the rear surface of recovered specimens after shock loading, (a) Vit 1 specimen, 

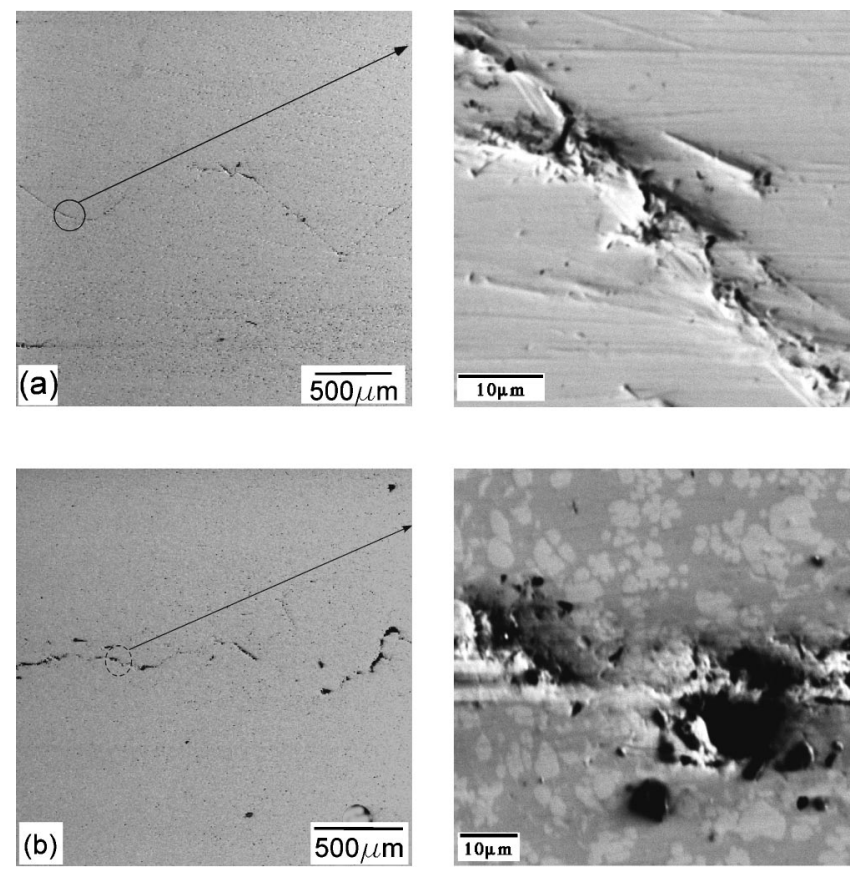

FIG. 4. Typical cross-section SEM micorgraphs of recovered specimens showing the damage area around the spall plane. (a) Microvoids nucleated within shear bands observed in Vit 1 specimen, (b) microvoids nucleated at the boundaries of $\beta$ phase (bright spots) and the matrix in $\beta$-Vit specimen.

the compression damage did occur during the compression, material strength played a dominant role, and, therefore the shock velocity increased with increasing particle velocity. When the internal damage increased to some degree, the weakening or softening of material became more important. Large portion of shock energy was expended in raising the temperature within the severely deformed shear bands or the generation of new microcracks, so in turn the shock velocity was being reduced. However, when the shock loading increased further, the rate of energy delivered into the shock front was much larger than that of the energy consumed by deformation and damage, the being damaged material was recompressed within shock front, therefore, higher shock velocity could be reached.

The spall strength of material can be estimated using the velocity pullback during the decompression process $(\Delta u$ in Fig. 1). ${ }^{12}$ Based on the measured deformation wave speed (Fig. 2), the calculated spall strengthes for Vit 1 and $\beta$-Vit are 2.35 and $2.11 \mathrm{GPa}$, respectively, and the estimated average strain rates corresponding to fastest rise portion of shock front are about $2 \times 10^{6} \mathrm{~s}^{-1}$. Comparing the spall strengths with the ultimate tensile limits at strain rate of $10^{-3} \mathrm{~s}^{-1}$, which are respectively 1.89 and $1.45 \mathrm{GPa}$, the two materials are only moderately sensitive to strain rate, with $\beta$-Vit composite exhibiting higher rate sensitivity.

The SEM micrographs of cross-sectioned recovered specimens for experiments No. 02 (Vit 1) and No. 09 ( $\beta$-Vit) are shown in Fig. 4. It is clearly seen that the spall damage mechanisms of the two materials are quite different. The zipshaped cracks on the micrographs of Vit 1 [Fig. 4(a)] suggest that the spalling of Vit 1 is characterized by brittle damage resulting from the shear localization even though the sample is under tension during decompression process. Examination under higher magnification [Fig. 4(a)] reveals that each crack is formed due to the nucleation, growth and coalescence of microvoids within the shear bands. While for $\beta$-Vit, its spalling is characterized to be much more ductile [Fig. 4(b)] and the damage seems to be due to the debonding induced by the tensile stress at phase boundaries exceeding the bond strength between the ductile bcc $\beta$ phase and the amorphous Vit 1 like matrix. It is clearly seen that there are many microvoids mostly nucleating at the boundaries separating the $\beta$ phase from the Vit 1 like matrix. The spall plane is formed in damage area due to the growth and coalescence of those microvoids.

In this letter, the shock responses of Vit 1 amorphous metallic glass and its composite, $\beta$-Vit, were investigated using planar impact technique. The experimentally obtained shock particle velocity profiles, shock $U_{s}-U_{p}$ Hugoniots, and the SEM examination of recovered specimen samples indicate a complex deformation process during shock compression. Inelastic deformation accompanying strong strain hardening starts at very low stress level due to the amplification of stress around the microscopic defects (the free volume spots) within the intact materials. Shear localization induced damage/failure, as well as the accompanying stress relaxation characterizes the subsequent compression process.

The authors thank Professor W. L. Johnson for providing the materials and many fruitful discussions. The work was supported by the DARPA's structural amorphous metals program through the Army Research Office (Grant No. DAAD \# 19-01-1-0525).

${ }^{1}$ W. L. Johnson, MRS Bull. 24, 42 (1999).

${ }^{2}$ A. Inoue, in Amorphous and Nanocrystalline Materials: Preparation, Properties and Applications, edited by A. Inoue and K. Hashimoto (Springer, Berlin, 2001), p. 1.

${ }^{3}$ A. Peker and W. L. Johnson, Appl. Phys. Lett. 63, 2342 (1993).

${ }^{4}$ C. C. Hays, C. P. Kim, and W. L. Johnson, Phys. Rev. Lett. 84, 2901 (2000).

${ }^{5}$ S. Zhuang, Ph.D. thesis, Caltech, 2002.

${ }^{6}$ J. R. Asay and M. Shahinpoor, High-Pressure Shock Compression of Solids (Springer, Berlin, 1993).

${ }^{7}$ L. M. Barker and R. E. Hollenbach, J. Appl. Phys. 43, 4699 (1972).

${ }^{8}$ R. G. McQueen, in High-Pressure Equations of State: Theory and Applications, Proceedings of the International School of Physics 《Enrico Fermi $\rangle$, Course CXIII, edited by S. Eliezer and R. A. Ricci (NorthHolland, Amsterdam, 1991), p. 101.

${ }^{9}$ M. E. Kipp and D. E. Grady, J. Phys. IV 4, 249 (1994).

${ }^{10}$ D. E. Grady, Mech. Mater. 29, 181 (1998).

${ }^{11}$ D. E. Grady, J. Phys. IV 4, 385 (1994).

${ }^{12}$ D. E. Grady and M. E. Kipp, High-Pressure Shock Compression of Solids (Springer, Berlin, 1993), p. 265. 